\title{
Hyaluronic acid-carboxymethylcellulose reduced postoperative bowel adhesions following laparoscopic urologic pelvic surgery: a prospective, randomized, controlled, single-blind study
}

U-Syn Ha ${ }^{1}$, Jun Sung Koh ${ }^{2,5^{*}}$, Kang Jun Cho ${ }^{2}$, Byung $\|$ Yoon $^{3}$, Kyu Won Lee ${ }^{4}$, Sung Hoo Hong ${ }^{1}$ and Ji Youl Lee ${ }^{1}$

\begin{abstract}
Background: To assess the anti-adhesive effect of treatment with hyaluronic acid-carboxymethylcellulose following laparoscopic radical prostatectomy.

Methods: This was a randomized, controlled, single-blind, parallel-group study using hyaluronic acid-carboxymethylcellulose in patients who underwent laparoscopic radical prostatectomy. Sixty patients were enrolled in the study. All patients were randomly assigned to either the hyaluronic acid-carboxymethylcellulose treatment group $(n=30)$ or the control group $(n=30)$. Viscera slide ultrasounds and plain X-rays were obtained at enrollment (V0), postoperative week $12(\mathrm{~V} 1)$, and $24(\mathrm{~V} 2)$. The primary end point was the difference in the excursion distance in the viscera slide ultrasound between V0 and V2.

Results: A total of 50 patients completed this study. The average excursion distance at V2 in the experimental group $(n=25)$ was significantly longer than in the control group $(n=25,2.7 \pm 1.2$ vs. $1.3 \pm 1.0 \mathrm{~cm}$, respectively; $p<0.001)$. The differences in the $\mathrm{V} 0$ and $\mathrm{V} 2$ excursion distances were significantly higher in the control group than in the experimental group $(1.48 \pm 1.5$ vs. $2.9 \pm 1.2 \mathrm{~cm}$, respectively; $p<0.001)$. None of patients showed adverse events associated with the use of hyaluronic acid-carboxymethylcellulose.
\end{abstract}

Conclusion: This randomized study demonstrated that hyaluronic acid-carboxymethylcellulose treatment resulted in a reduction in bowel adhesion to the abdominal wall after laparoscopic pelvic surgery and had good clinical safety.

Trial registration: ClinicalTrials.gov Identifier: NCT02773251 Date: May 12, 2016.

Keywords: Postoperative adhesion, Laparoscopy, Adhesion barrier

\footnotetext{
* Correspondence: gostraight@catholic.ac.kr

Department of Urology, Bucheon St. Mary's Hospital, College of Medicine,

The Catholic University of Korea, Seoul, Republic of Korea

${ }^{5}$ Department of Urology, Bucheon St. Mary's Hospital, College of Medicine,

The Catholic University of Korea, 327, Sosa-ro, Wonmi-gu, Bucheon-si,

Gyeonggi-do 14647, Republic of Korea

Full list of author information is available at the end of the article
} 


\section{Background}

Postoperative adhesions frequently occur following abdominal surgery $[1,2]$. Peritoneal adhesions are a consequence of surgical trauma such as dissection, cutting, and coagulation, and can result in adhesion-related complications that can increase health care costs. To date, there no effective treatments for adhesions have been developed. Thus, the prevention and reduction of adhesions is the best management strategy [3].

Many researchers have been trying to find effective methods to prevent adhesions, and various barrier materials have been developed and studied. Individual studies with barrier materials have reported positive results in the prevention of postoperative adhesions $[4,5]$. However, another study on barriers did not demonstrate efficacy in reducing adhesions [6]. A meta-analysis from 28 trials (5191 patients) reported that oxidized regenerated cellulose and Hyaluronic acid-carboxymethylcellulose (HA/CMC) can safely reduce the clinically relevant consequences of adhesions [7]. Most of the trial agents evaluated in the above studies were based on open bowel surgery. Recently, laparoscopic surgery has been expanding rapidly and has gained acceptance as a viable alternative to traditional open surgery [8]. A certain degree of peritoneum loss should be also inevitable during laparoscopic pelvic surgery (i.e., laparoscopic radical prostatectomy and cystectomy), although the loss of peritoneum should be smaller than open surgery.

There are a few studies based on patients who have undergone laparoscopic surgery $[5,9,10]$, but no study has targeted laparoscopic urologic surgery. In addition, these studies did not directly assess the presence of adhesions. The purpose of this study was to assess the presence of adhesions as determined by viscera slide ultrasound after treatment with HA/CMC following laparoscopic radical prostatectomy.

\section{Methods}

This was a prospective, randomized, controlled, singleblind, parallel-group study using HA/CMC (marketed as Guardix-sol ${ }^{\oplus}$, Hanmi Medicare, Seoul, Korea) in patients who underwent laparoscopic radical prostatectomy between November 2011 and June 2014. All the patients were informed in detail about the aims and the procedures of the study and they signed a written informed consent prior inclusion into the study. The protocol and the written informed consent were approved by the local ethical committee (Catholic Medical Center, Clinical Research Coordinator Center; approval number XC11DIMI10098H).

\section{Subjects}

Men who were 50-75 years old and diagnosed with prostate cancer were eligible if they were scheduled to undergo laparoscopic radical prostatectomy. Exclusion criteria included any history of abdominal or pelvic surgery, hypersensitivity or an allergic reaction to the study material, pelvic lymph node dissection at the same time as prostatectomy, the presence of surgical site infection or contamination, a history of a medical disease causing bowel adhesion, or a history of severe drug allergies.

\section{Study design and protocol}

The laparoscopic radical prostatectomy was performed in same surgical procedures and steps by two surgeons (USH and JSK) who have experienced over 150 cases of laparoscopic radical prostatectomy. The laparoscopic radical prostatectomy was performed using the fiveport fan-shaped transperitoneal approach. After the introducing the peritoneal cavity, incising the parietal peritoneum between the medial umbilical ligaments are incised and dissection is carried through the fatty alveolar tissue to develop the space of Retzius. After that, the surgical steps are following order (1) incision of the endopelvic fascia; (2) ligation of the dorsal vein complex; (3) division of the bladder neck; (4) dissection of the seminal vesicles; (5) incision of the Denonvillier fascia and control of the lateral pedicles with antegrade neurovascular bundle dissection; (8) apical dissection and division of the dorsal vein and the urethra; (9) urethrovesical anastomosis.

Considering about $30 \%$ of dropout rate (under the assumption of $40 \%$ difference between HA/CMC treatment group and the control group based on previous similar study ${ }^{10}$ ), by which the target enrollment for this trial was 60 subjects (30 subjects per group). The sample size was determined assuming a level of significance of $\alpha=0.05$ (two-side) and a $80 \%$ statistical power of test. All patients were randomly assigned to either the $\mathrm{HA} / \mathrm{CMC}$ treatment group $(n=30)$ or the control group $(n=30)$ using a computer-generated randomization table. The surgeon was blinded to treatment assignments before randomization. Patients were also blinded to their treatment group throughout the study. HA/ CMC was applied in all port sites and the peritoneal incision line of the medial umbilical ligament with a single-use applicator attached to a sprayer that allowed for the precise application to the required sites (Fig. 1). The amount of HA/CMC applied was $5 \mathrm{ml}$. Information regarding the duration of illness and medical history were collected at the time of enrollment (V0). Viscera slide ultrasound and plain $\mathrm{X}$-ray were recorded at the time of the operation (V0) and 12 (V1) and 24 week (V2) after the operation.

The primary end point was the difference in excursion distance on viscera slide ultrasound between V0 and V2. The secondary end point was excursion distance on 


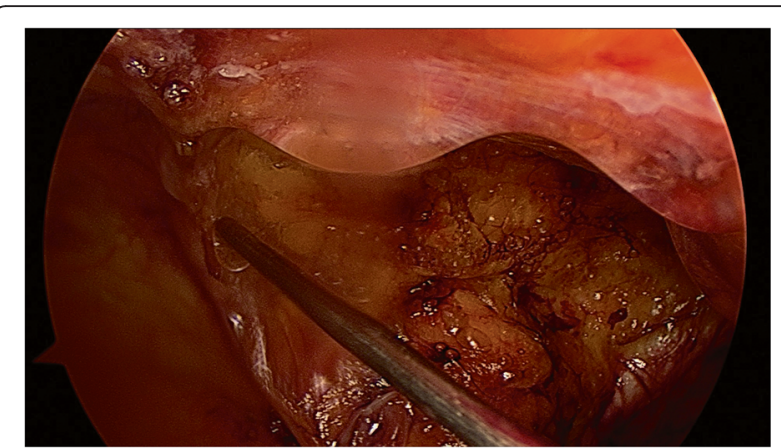

Fig. 1 A view of HAVCMC application: HA/CMC was applied to the port site and peritoneal incision line of the medial umbilical ligament with a single-use applicator

viscera slide ultrasound at V2 and the presence of restriction of viscera slide on ultrasound at V2.

\section{Assessment of efficacy and safety}

Twelve and 24 weeks after the operation, bowel adhesion to the abdominal wall was evaluated by ultrasound and plain X-rays. We performed viscera slide ultrasound according to a technique that has been previously described [11]. By dividing the abdomen into 5 segments and examining the viscera slide in each segment, a prediction of the extent of the adhesions was made for each patient. Figure 2 shows the division of the abdomen into 5 segments and their numbering. At the time of the viscera slide ultrasound, data were also collected on the location of the scars on the abdomen. The main point of interest was the distance of the longitudinal

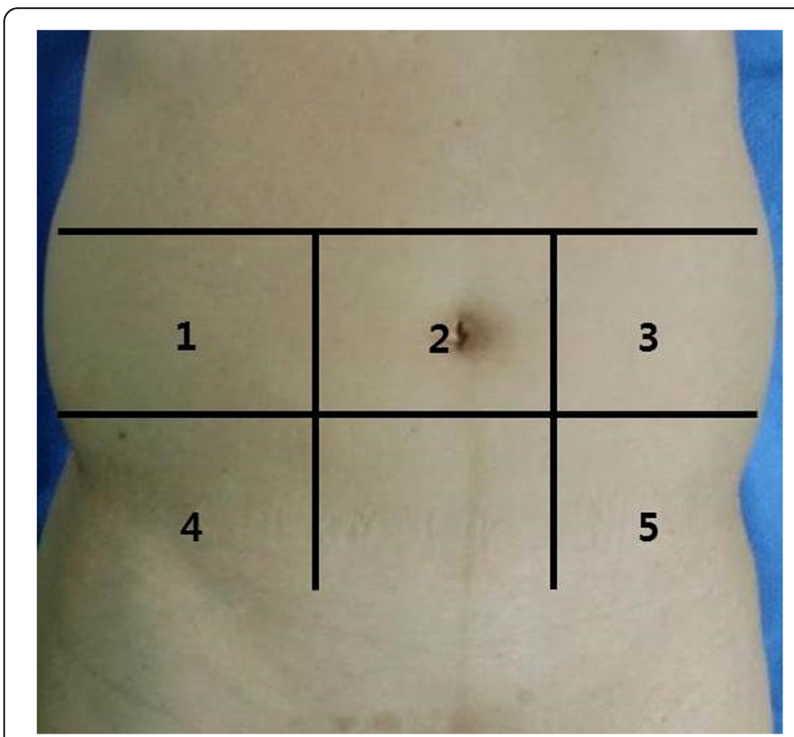

Fig. 2 Map of the abdomen divided into 5 segments by bilateral, vertical, and proximal one-third clavicular lines, a transverse line across the supraumbilical region, and a transverse line across the anterior superior iliac spines excursion of the selected area in relation to the fixed abdominal wall. Normal viscera sliding movement was defined as equal to or greater than $1 \mathrm{~cm}$ of longitudinal movement. Restricted viscera slide was defined as less than $1 \mathrm{~cm}$ of longitudinal movement during both normal and exaggerated respiration. The ultrasound was performed by two sonographers who had been well instructed for study assessment. The assessment by ultrasound was double-checked. The sonographer, radiologist and all accessor was blind to the randomization during the all study period.

\section{Statistical analysis}

The data for this study are expressed as mean \pm standard deviation of the mean. The comparisons of the 2 groups were made using a $X^{2}$ test, an independent Student's $t$ test, or repeated measure ANOVA. $P$-values $<0.05$ were considered significant. Statistical calculations were carried out with IBM SPSS statistics, Version 21 (IBM Corp, Armonk, NY).

\section{Results}

A total of 60 patients who diagnosed with prostate cancer were enrolled and 50 patients completed this study. In the HA/CMC group, two patients were lost to followup, two patients dropped out because they don't want to undergo a sonography test and one patient was switched to open radical prostatectomy. In the control group, two patients were lost to follow-up and three patient dropped out because they don't want to undergo a sonography test. The characteristics of the patients who completed the study are summarized in Table 1 . There were no statistically significant differences in the baseline characteristics between groups. There were also no differences in the perioperative findings between groups (Table 2), nor were there any differences in the number of ports used for each patient (5 ports), the size of the ports $(1.1 \mathrm{~cm})$, or the site of insertion. None of the patients enrolled in this study showed postoperative complications such as wound infections, bladder urethral anastomosis leakage, post-operative ileus, or adverse events (e.g. hypersensitivity or an allergic reaction) associated with the use of HA/CMC.

Table 3 shows the results of the adhesion characteristics in the experimental and control groups. The average post-operative excursion distance in the experimental group $(2.7 \pm 1.2 \mathrm{~cm})$ was significantly longer than that of the control group $(1.3 \pm 1.0 \mathrm{~cm} ; p<0.001)$. The differences in the V0 and V2 excursion distances were significantly higher in the control group $(2.9 \pm 1.2 \mathrm{~cm})$ than in the experimental group $(1.48 \pm 1.5 \mathrm{~cm} ; p<0.001)$.

According to the restriction criteria, a total of 43 sites showed visceral slide restriction in the experimental group, while a total of 74 sites in the control group 
Table 1 Demographic data of the Hyaluronic acidcarboxymethylcellulose (HA/CMC) group and the control group

\begin{tabular}{|c|c|c|c|}
\hline & $\begin{array}{l}\text { HA/CMC group } \\
(n=25)\end{array}$ & $\begin{array}{l}\text { Control group } \\
(n=25)\end{array}$ & $p$-value \\
\hline Age (years), mean $\pm S D$ & $67.5 \pm 9.3$ & $65.4 \pm 10.5$ & 0.542 \\
\hline BMI $\left(\mathrm{kg} / \mathrm{m}^{2}\right)$, mean $\pm \mathrm{SD}$ & $24.7 \pm 2.9$ & $25.1 \pm 2.6$ & 0.453 \\
\hline \multicolumn{4}{|l|}{ Combined disease, $n(\%)$} \\
\hline DM & 6 & 4 & 0.480 \\
\hline Cardiovascular dis. & 8 & 7 & 0.758 \\
\hline $\begin{array}{l}\text { Gastrointestinal dis., } \\
\text { hepatitis }\end{array}$ & 12 & 10 & 0.569 \\
\hline \multicolumn{4}{|l|}{ Smoking history } \\
\hline Current smoker & 10 & 12 & 0.569 \\
\hline Non-smoker & 15 & 13 & \\
\hline \multicolumn{4}{|l|}{$\begin{array}{l}\text { ASA physical status } \\
\text { classification }\end{array}$} \\
\hline 1 & 14 & 17 & 0.382 \\
\hline 2 & 11 & 8 & \\
\hline $3-6$ & 0 & 0 & \\
\hline
\end{tabular}

$B M I$ body mass index, DM diabetes mellitus, ASA American Society of Anesthesiologists

showed restriction, which was a significant difference. Plain X-rays showed an ileus gas pattern in patients in both groups (experimental group: $8 \%$ [2/25], control group: $16 \%$ [4/25]), but none of the participants complained of abdominal pain.

\section{Discussion}

The main findings of this study are that HA/CMC treatment increased bowel excursion distance in patients after laparoscopic pelvic surgery, suggesting that it reduced and prevented bowel adhesion to the damaged layer between anterior abdominal wall and peritoneum including port site. Furthermore, there were no reports of complications associated with the use of HA/CMC.

The key sites of adhesion formation are the port sites and damaged surface lining of the peritoneum between the medial umbilical ligaments. The peritoneal injury between anterior abdominal wall and anterior peritoneum,

Table 2 Operative and post-operative clinical data in the Hyaluronic acid-carboxymethylcellulose (HA/CMC) group and the control group

\begin{tabular}{llll}
\hline & $\begin{array}{l}\text { HA/CMC group } \\
(n=25)\end{array}$ & $\begin{array}{l}\text { Control group } \\
(n=25)\end{array}$ & $p$-value \\
\hline Pathological stage & & & \\
$\quad$ II & 21 & 18 & 0.306 \\
III & 4 & 7 & \\
IV & 0 & 0 & \\
Total operative surgical time & $214.7 \pm 82.5$ & $202.1 \pm 92.6$ & 0.212 \\
Estimated blood loss (ml) & $158.8 \pm 120.7$ & $173.9 \pm 113.5$ & 0.382 \\
\hline
\end{tabular}

which is inevitable, can cause a local inflammatory reaction with fibrous exudate and fibrin formation. Various factors can lead to postoperative adhesions, including the type and technique of surgery, individual predisposing factors, thermal injury, trauma, and a history of previous surgery. The balance between fibrin deposition and degradation is crucial in determining whether normal peritoneal healing or adhesion formation occurs, with peritoneal injury promoting an imbalance in fibrin kinetics which may serve as a scaffold for fibroblasts and capillary in-growth that form peritoneal adhesions [12]. Keeping peritoneal surfaces separate for peritoneal re-epithelialization is critical for preventing and decreasing adhesion.

Adhesive strength that can withstand gravity is considered to be an important factor for measuring success because the target site in this study is the abdominal wall. Park et al. conducted a study using Seprafilm (a hyaluronate/carboxycellulose-based membrane) and found that this membrane type was brittle and difficult to apply, with liquid devices likely to be more useful in this setting [13]. Another animal study showed that a liquid device appeared to be superior to Seprafilm ${ }^{\circ}$ [14]. The idea of using HA/CMC gel to reduce bowel adhesions to the abdominal wall was based on its relatively high adhesive strength, and previous studies have demonstrated a significant reduction of post-surgical adhesions after the instillation of HA/CMC solutions as tissue barriers during the healing process [15-17]. $\mathrm{HA} / \mathrm{CMC}$, which is a liquid-type synthetic physical sol-gel barrier with a viscosity ranging from 2500 to $3500 \mathrm{cP}$, is an anionic polysaccharide that is composed of D-glucuronic acid and N-acetyl-D-glucosamine [15]. Thus, HA/CMC is sticky and coats the peritoneum for a sufficient period and make up damage when it is sprayed on the tissue [18]. In addition to physical barrier to maintain a space, $\mathrm{HA} / \mathrm{CMC}$ can reduce inflammation by preventing the migration of leukocyte and fibroblasts to the operation site. Sohn et al showed in his experimental study that HA/CMC treatment significantly decrease average degrees of polymorphonuclear leukocyte and myofibroblasts infiltration [19].

In the present study, our results showed significantly increased bowel excursion distance in the treatment group as compared to the control group. The fact that patients treated with HA/CMC had reduced postoperative bowel adhesions to the abdominal wall can be attributed to the extended presence of the barrier and the maintenance of coating activity between the abdominal wall and bowel.

One distinctive feature of this study is its examination of the prevention of adhesions in laparoscopic pelvic surgery in the urologic field. This study is the first clinical report to evaluate the efficacy of strategies to 
Table 3 Adhesion characteristics in the Hyaluronic acid-carboxymethylcellulose (HA/CMC) group and the control group

\begin{tabular}{|c|c|c|c|c|c|c|c|}
\hline & \multicolumn{3}{|c|}{ HA/CMC group $(n=25)$} & \multicolumn{3}{|c|}{ Control group $(n=25)$} & \multirow[t]{2}{*}{$p$-value } \\
\hline & V0 (0 week) & V2 (24 weeks) & $p$-value & V0 (0 week) & V2 (24 weeks) & $p$-value & \\
\hline \multicolumn{8}{|l|}{ Ultrasound findings } \\
\hline Average excursion distance of the viscera slide & $4.2 \pm 0.6$ & $2.7 \pm 1.2$ & $<0.001^{\mathrm{a}}$ & $4.1 \pm 0.7$ & $1.3 \pm 1.0$ & $<0.001^{\mathrm{a}}$ & \\
\hline Difference in $\mathrm{V} 0$ and $\mathrm{V} 2$ & $1.48 \pm 1.5$ & & & $2.9 \pm 1.2$ & & & $<0.001^{\mathrm{b}}$ \\
\hline$\%$ of restricted viscera slide sites & 0 & $34.4(43 / 125)$ & & 0 & $59.2(74 / 125)$ & & $<0.001^{\mathrm{c}}$ \\
\hline
\end{tabular}

${ }^{\mathrm{a} i n d e p e n d e n t} t$-test, ${ }^{\mathrm{b}}$ repeated measure ANOVA, ${ }^{\mathrm{c}}{ }^{2}$ test

prevent adhesions to the abdominal wall, such as at the port site and the incision line of the medial umbilical ligament, in patients who have undergone laparoscopic radical prostatectomy. Most previous studies evaluating the use of HA/CMC have been conducted with open surgery. The fact that minimally invasive surgeries such as laparoscopic or robot-assisted laparoscopic surgery have become more popular in pelvic surgery without bowel resection and manipulation could affect the efficacy of HA/CMC as compared to previous studies. Thus, we conducted a study targeting patients who have undergone laparoscopic radical prostatectomy.

Another unique feature of this study is that we used ultrasonic detection and mapping of abdominal wall adhesions as an evaluation method, which was developed by Sigel B et al [11]. Although adhesions are the main cause of small bowel obstruction and ileus, postoperative adhesions do not always result in bowel obstruction, especially when pelvic surgery is performed without bowel resection or manipulation. Bowel adhesion to the abdominal wall is expected to be one of the most frequent sites of adhesion in patients who have undergone surgery without bowel resection or manipulation. This means that the presence of adhesions cannot be fully assessed by plain $\mathrm{x}$-ray or by clinical history in patients who have undergone surgery without bowel resection or manipulation. Ultrasound examination is a specific and reliable method to identify and detect adhesion-free areas [20, 21]. Previous studies have relied on second-look operations for the evaluation of post-operative adhesions, which is invasive and cannot be used to evaluate all patients who have had surgery. We selected ultrasound as an evaluation method to specifically evaluate adhesions between the abdominal wall and bowel.

This study had a relatively small sample size, resulting in limitation of its statistical power. Another initial concern was a lack of interest among patients to be enrolled in the control group because many wanted to be treated with HA/CMC. These data and results must be considered as a preliminary report, although the fact that these findings were statistically significant should be noted.

\section{Conclusions}

This randomized study provided preliminary data demonstrating that $\mathrm{HA} / \mathrm{CMC}$ treatment resulted in a reduction in bowel adhesion to the abdominal wall after laparoscopic pelvic surgery with good clinical safety.

\section{Abbreviation}

HA/CMC, hyaluronic acid-carboxymethylcellulos

\section{Acknowledgements}

We would like to acknowledge the support provided by all the staff of the Department of Urology, The Catholic University of Korea, Seoul, Korea. We also thank the Hanmi Medicare for providing HA/CMC.

\section{Funding}

The study was funded by Hanmi Medicare, Seoul, Korea. The funding sources had no role in study design; in the collection, analysis and interpretation of data; in the writing of the report; and in the decision to submit the article for publication.

\section{Availability of data and materials}

Raw dataset from the sample cannot be shared since the informed consent from the participants at the time of the study recruitment only included the publication of the study results but not sharing them in public.

\section{Authors' contributions}

USH and JSK participated in the study design. KJC, BIY, SHH, KWL and JYL acquired the data. JSK analysed and interpreted the data. USH and KJC drafted the manuscript. JSK and JYL critically revised the manuscript for important intellectual content. All authors read and approved the final manuscript.

Competing interests

The authors declare that they have no competing interests.

\section{Consent for publication}

The informed consent included the agreement of publication of any pictures in anonymity of the patient.

\section{Ethics approval and consent to participate}

All the patients were informed in detail about the aims and the procedures of the study and they signed a written informed consent prior inclusion into the study. The protocol and the written informed consent were approved by the local ethical committee (Catholic Medical Center, Clinical Research Coordinator Center; approval number XC11DIMI10098H).

\section{Author details}

${ }^{1}$ Department of Urology, Seoul St. Mary's Hospital, College of Medicine, The Catholic University of Korea, Seoul, Republic of Korea. ${ }^{2}$ Department of Urology, Bucheon St. Mary's Hospital, College of Medicine, The Catholic University of Korea, Seoul, Republic of Korea. ${ }^{3}$ Department of Urology, Catholic Kwandong University, International St. Mary's Hospital, Incheon, Republic of Korea. ${ }^{4}$ Department of Urology, St. Paul's Hospital, College of Medicine, The Catholic University of Korea, Seoul, Republic of Korea.

${ }^{5}$ Department of Urology, Bucheon St. Mary's Hospital, College of Medicine, 
The Catholic University of Korea, 327, Sosa-ro, Wonmi-gu, Bucheon-si, Gyeonggi-do 14647, Republic of Korea.

Received: 4 February 2016 Accepted: 3 June 2016 Published online: 10 June 2016

\section{References}

1. Becker JM, Dayton MT, Fazio WW, Beck DE, Stryker SJ, Wexner SD, et al. Prevention of postoperative abdominal adhesions by a sodium hyaluronatebased bioresorbable membrane: a prospective, randomized, double-blind multicenter study. J Am Coll Surg. 1996;183:297-306.

2. Menzies $\mathrm{D}$, Ellis $\mathrm{H}$. Intestinal obstruction from adhesions-how big is the problem? Ann R Coll Surg Engl. 1990;72:60-3.

3. Diamond MP, Wexner SD, diZereg GS, Korell M, Zmora O, Van Goor H, et al. Adhesion prevention and reduction: current status and future recommendations of a multinational interdisciplinary consensus conference. Surg Innov. 2010;17:183-8.

4. Larsson B, Lalos O, Marsk L, Tronstad SE, Bygdeman M, Pehrson S, et al. Effect of intraperitoneal instillation of $32 \%$ dextran 70 on postoperative adhesion formation after tubal surgery. Acta Obstet Gynecol Scand. 1985;64:437-41.

5. Takeuchi H, Kitade M, Kikuchi I, Shimanuki H, Kumakiri J, Kinoshita K. Adhesion-prevention effects of fibrin sealants after laparoscopic myomectomy as determined by second-look laparoscopy: a prospective, randomized, controlled study. J Reprod Med. 2005;50:571-7.

6. Tang CL, Jayne DG, Seow-Choen F, Ng YY, Eu KW, Mustapha N. A randomized controlled trial of $0.5 \%$ ferric hyaluronate gel (Intergel) in the prevention of adhesions following abdominal surgery. Ann Surg. 2006;243:449-55.

7. ten Broek RP, Stommel MW, Strik C, van Laarhoven CJ, Keus F, van Goor H. Benefits and harms of adhesion barriers for abdominal surgery: a systematic review and meta-analysis. Lancet. 2014;383:48-59.

8. Mettler L. Pelvic adhesions: laparoscopic approach. Ann N Y Acad Sci. 2003; 997:255-68.

9. Fossum GT, Silverberg KM, Miller CE, Diamond MP, Holmdahl L. Gynecologic use of Sepraspray Adhesion Barrier for reduction of adhesion development after laparoscopic myomectomy: a pilot study. Fertil Steril. 2011;96:487-91.

10. Tchartchian G, Hackethal A, Herrmann A, Bojahr B, Wallwiener C, Ohlinger R, et al. Evaluation of SprayShield ${ }^{\mathrm{TM}}$ Adhesion Barrier in a single center: randomized controlled study in 15 women undergoing reconstructive surgery after laparoscopic myomectomy. Arch Gynecol Obstet. 2014;290:697-704.

11. Sigel B, Golub RM, Loiacono LA, Parsons RE, Kodama I, Machi J, et al. Technique of ultrasonic detection and mapping of abdominal wall adhesions. Surg Endosc. 1991;5:161-5.

12. Arung $W$, Meurisse $M$, Detry O. Pathophysiology and prevention of postoperative peritoneal adhesions. World J Gastroenterol. 2011;17:4545-53.

13. Park CM, Lee WY, Cho YB, Yun HR, Lee WS, Yun SH, et al. Sodium hyaluronate-based bioresorbable membrane (Seprafilm) reduced early postoperative intestinal obstruction after lower abdominal surgery for colorectal cancer: the preliminary report. Int J Colorectal Dis. 2009;24:305-10.

14. Oncel M, Remzi FH, Senagore AJ, Connor JT, Fazio WW. Comparison of a novel liquid (Adcon-P) and a sodium hyaluronate and carboxymethylcellulose membrane (Seprafilm) in postsurgical adhesion formation in a murine model. Dis Colon Rectum. 2003;46:187-91.

15. Hong JH, Choe JW, Kwon GY, Cho DY, Sohn DS, Kim SW, et al. The effects of barrier materials on reduction of pericardial adhesion formation in rabbits: a comparative study of a hyaluronan-based solution and a temperature sensitive poloxamer solution/gel material. J Surg Res. 2011;166:206-13.

16. Leach RE, Burns JW, Dawe EJ, SmithBarbour MD, Diamond MP. Reduction of postsurgical adhesion formation in the rabbit uterine horn model with use of hyaluronate/carboxymethylcellulose gel. Fertil Steril. 1998;69:415-8.

17. Lee JM, Baek S. Antiadhesive effect of mixed solution of sodium hyaluronate and sodium carboxymethylcellulose after blow-out fracture repair. J Craniofac Surg. 2012;23:1878-83.

18. Kimmelman CP, Edelstein DR, Cheng HJ. Sepragel sinus (hylan B) as a postsurgical dressing for endoscopic sinus surgery. Otolaryngol Head Neck Surg. 2001;125:603-8.

19. Sohn EJ, Ahn HB, Roh MS, Ryu WY, Kwon YH. Efficacy of temperature-sensitive Guardix-SG for adhesiolysis in experimentally induced eyelid adhesion in rabbits. Ophthal Plast Reconstr Surg. 2013;29:458-63.

20. Zinther NB, Zeuten A, Marinovskij E, Haislund M, Friis-Andersen H. Detection of abdominal wall adhesions using visceral slide. Surg Endosc. 2010;24:3161-6.
21. Kolecki RV, Golub RM, Sigel B, Machi J, Kitamura H, Hosokawa T, et al. Accuracy of viscera slide detection of abdominal wall adhesions by ultrasound. Surg Endosc. 1994:8:871-4.

\section{Submit your next manuscript to BioMed Central and we will help you at every step:}

- We accept pre-submission inquiries

- Our selector tool helps you to find the most relevant journal

- We provide round the clock customer support

- Convenient online submission

- Thorough peer review

- Inclusion in PubMed and all major indexing services

- Maximum visibility for your research

Submit your manuscript at www.biomedcentral.com/submit 